\title{
Medical Students' Career Choice and Attitudes towards Family Medicine in Morocco
}

\author{
Majda Sebbani (iD, ${ }^{1,2}$ Adil Mansouri, ${ }^{1,2}$ Latifa Adarmouch,, ${ }^{1,2}$ and Mohamed Amine ${ }^{1,2}$ \\ ${ }^{1}$ Community Medicine and Public Health Department, Research Laboratory, Biosciences and Health, School of Medicine, \\ Cadi Ayyad University, Marrakech, Morocco \\ ${ }^{2}$ Clinical Research Unit, Mohammed VI University Hospital, Marrakech, Morocco
}

Correspondence should be addressed to Majda Sebbani; dr.sebbani@gmail.com

Received 22 June 2020; Revised 22 July 2020; Accepted 3 August 2020; Published 28 August 2020

Academic Editor: Haoran Xie

Copyright (c) 2020 Majda Sebbani et al. This is an open access article distributed under the Creative Commons Attribution License, which permits unrestricted use, distribution, and reproduction in any medium, provided the original work is properly cited.

\begin{abstract}
Background. The motivation of this work is driven on the one hand from the need to understand the medical students' attitude towards medical training in the context of the reform in Morocco and the creation of "family medicine" as a specialty. This study aims to explore the expectations of medical students regarding family medicine and to identify the factors that may influence setting their choices after graduation. Methods. We conducted a cross-sectional study among Moroccan medical students from public faculties during the month of August 2019. The data collection was based on an online self-administered electronic questionnaire. The quantitative data were analyzed by SPSS version 16. The analyses were descriptive univariate (Fisher's test) and multivariate (binary logistic regression) with a $P$ value of $5 \%$. The qualitative data were synthesized according to a thematic analysis grid. Results. The medical students in Morocco have a positive perception of family medicine as an important specialty but low interest in it as a future career. Only $6.4 \%$ had the intention to choose it as a future career. However, $27.5 \%$ chose to become a general practitioner if it is a specialty (family medicine, as part of the new medical reform). The factors associated with the choice of career in general practice were mainly the ambition for career development $(\mathrm{OR}=4.8 ; 95 \% \mathrm{CI}[2.46$; 9.51]), income $(\mathrm{OR}=2.6$; $95 \%$ CI $[1.11 ; 6.29])$, or the personal experience as a student or patient in contact with a general practitioner $(\mathrm{OR}=0.48$; $95 \% \mathrm{CI}$ $[0.25 ; 0.92])$. Conclusion. The practical experience with family medicine seems to have an important influence on being attracted to family medicine careerwise. The study findings will inform future planning to introduce the residency program.
\end{abstract}

\section{Introduction}

A good health system requires a strong primary health care through the management of its financial and human resources as well as organizational capacities. Also, a comprehensive approach to health and well-being centered on the needs and preferences of individuals, families, and communities is a necessity [1]. Human resources' challenges in developing countries are not only quantitative but also qualitative. The World Health Organization (WHO) recommends having 23 qualified personnel per 10,000 inhabitants in order to achieve the Millennium Development Goals. Morocco is very far in medical density compared to neighboring countries (7.27/10,000 inhabitants in 2016 compared to 18 in Algeria, 12.7 in Tunisia, and 40.69 in
Spain) [2]. Morocco is suffering from a significant shortage in human resources in primary care, particularly of general practitioners (GPs) who provide continuous monitoring and care. According to the last situation in 2016, the number of doctors is 25,207 with only $36 \%$ who are general practitioners [3]. To meet WHO standards, the need for GPs is estimated at 3,829 .

Rectifying this situation requires not only an increase in the recruitment of general practitioners, but also an improvement in the skills of these doctors and the general conditions of their training. This vision, which has been widely adopted internationally by adapting training to the real current and future needs of populations, has given birth to a new professional identity named "general or family medicine." General practice or family medicine as defined by 
WONCA in 2002 (European Society of General MedicineFamily Medicine) is "an academic and scientific discipline, with its own educational content, research, evidence base and clinical activity, and a clinical specialty orientated to primary care" [4].

The Moroccan reform of medical studies thus highlighted the creation of a specialization branch in family medicine [5]. This section of the reform sparked a broad discussion on the development and creation of a family medicine specialty from the point of view of its objectives, methods, and differences with general medicine. The current undergraduate medical curriculum lasts 7 years and allows students to obtain a medical degree in general medicine after the medical thesis. These graduates can then either settle in private to practice general medicine or pass a hiring contest to practice general medicine in the public sector. The other option is to enroll in residency program, through a contest, to become a specialist (4 years for medical specialties and 5 years for surgical ones). Another contest is open to students in the 6th year to recruit a limited number of interns who will be trained at the university hospital for 2 years before enrolling in a residency program. In fact, the choice of career is strongly influenced by the experience and attitude towards the specialty. This link has been proven in the literature by several authors in different contexts and medical education programs [6-8].

The assessment of the attitude of Moroccan students towards primary care practice in general medicine and their perspectives for choosing general medicine/family medicine are important. From a qualitative point of view, it is necessary to take into consideration the question of motivation and that of the working conditions and career prospects, allowing the retention of personnel in primary care. Faced with this double challenge of doctor shortage and skill requirement and with the imperative of improving the recruitment and quality of primary care physicians, it is essential to approach their perceptions of practice and their prospects for career choice.

At the end of this study, which aims to assess the attitudes and perceptions of Moroccan student doctors and factors associated with the choice of general medicine as a specialty, we can make recommendations to improve recruitment in general medicine and subsequently prepare the choice of family medicine specialty.

\section{Methods}

We conducted a cross-sectional survey of medical students from public medical schools in Morocco. We explored the students' career prospects and their attitudes towards the choice of general medicine/family medicine.

The questionnaire developed from the literature review included close-ended questions: dichotomous, multiple choice, and Likert-scale score with level 1 (strongly disagree), 2 (somewhat disagree), 3 (neutral), 4 (somewhat agree), 5 (strongly agree), and open-ended questions. It was distributed online and was sent after validation and testing with a group of students. Data were collected during August and September 2019. Students in the 1st cycle (1st and 2 nd year of medicine) or 2nd cycle (from the 3rd year until obtaining the medical degree) were invited to participate through social networks and student associations of public Moroccan faculties. The survey was opened for 2 months with 2 reminders of participation. We collected sociodemographic data (level of study, gender, family situation, level of education and profession of the spouse, presence of doctors among the family-parents, brothers, and sisters), the choice of specialty after graduation (medical, surgical, biology, or general medicine/family medicine), attitude towards primary care, and factors associated with the choice of career in general medicine/family medicine (factors influencing the attitude of students). Participants were asked about their career choice through the two following questions:

(1) By giving the first career choice after their graduation (general medicine was in the list of the possible choices)

(2) Their perspective of choosing general medicine if it becomes a "specialty = family medicine"

The theoretical model adopted was inspired by the Eagly and Chaiken model adapted by Emma Henderson [9, 10]. Factors influencing attitudes towards general medicine and the practice of general practitioners were gender, educational level, the role model (experience in contact with a general practitioner), the influence of the family (parents, brothers, or medical spouses), the media, the income, the motivation, and the self-perceived clinical and psychosocial skills.

The responses were extracted by Excel, coded and then analyzed using SPSS software (V16). The statistical analyses were descriptive and univariate. Qualitative variables were presented as numbers and percentages, and quantitative variables were presented as means and standard deviations SD (shown from mean values of Likert-scale values \pm SD). Fisher's exact test compared two proportions from two independent samples. The comparison of the scores means obtained for the attitudes and perceptions of students according to gender (male and female) and to study level (1st and 2nd cycle) used the nonparametric Wilcoxon-Mann-Whitney test.

The study of the factors associated with the choice of career in family medicine was carried out in two stages: (1) in univariate analysis by the Fisher Exact test and (2) in multivariate analysis using binary logistic regression to model the factors influencing the choice of family medicine as a specialty. The Forward Stepwise method with 5\% entry and 20\% exit thresholds. The Hosmer and Lemeshow test made it possible to test the final model retained after adjustment for the different significant factors in a multivariate analysis. The significance level for all statistical analyses was $5 \%$.

The answers to the open-ended questions were analyzed separately according to a content analysis guide and then according to the quantitative results. Confidentiality and anonymity were respected during the data collection and analysis. Participation was free based on the principle of volunteering. 


\section{Results}

3.1. Characteristics of the Participating Students. The number of respondents was 280. Female to male sex ratio was 2.11. Participants at the end ( 7 th year) or at the first year of medical training were the most predominant $(22.1 \%$ and $14.6 \%$, resp.). Students belonging to the schools of "Marrakech" and "Casablanca" represented the majority (57\%) (Table 1).

3.2. Career Prospects and Choice of General Medicine. Only $6.4 \%$ chose "general medicine" as a career after graduation. Figure 1 shows the proportions of the responses compared to the choice of specialty (only one choice per participant): $47.1 \%$ answered medical specialty, $43.2 \%$ surgical specialty, and $3.2 \%$ specialty in biology. Cardiology and general surgery were the most desired (Figure 1).

3.3. Attitudes and Perspectives towards Primary Care and General Medicine according to Gender and Study Level. Analysis of the data by gender and by study cycle revealed a difference in some questions relating to attitudes and perceptions towards general medicine (Table 2). The students had positive attitudes towards general practice and thought that general medicine is a valuable discipline like the other specialties (average Likert score $=3.62 / 5$ ). This result was more significant in graduate students $(P<0.0001)$. They felt that the experiences on the primary care were rewarding regardless of the career choice. But compared to the work of a specialist, the answers were against general medicine, especially among undergraduates. These students disagreed with choosing general medicine as an attractive career option compared to their elders $(P=0.003)$. As for the influence of gender, women placed more importance on prevention and psychosocial aspects than men $(P<0.001$ and $P=0.001$, resp.). The perception of graduate students regarding the place of the general practitioner in the health system was more positive.

3.4. Factors Influencing the Choice of Specialty in Family Medicine. Almost a third (27.5\%) chose to become a general practitioner (GP) if it becomes a specialty (family medicine, as part of the new medical reform). The factors associated with a career in general medicine were mainly personal experience as a student or patient in contact with a general practitioner (58.4\%, $P<0.0001$ and $45.5 \%, P=0.004$ ), the ambition of career development $(45.5 \%, P<0.0001)$, the self-perceived quality of human and psychosocial skills $(55.8 \%, P<0.0001)$, and the opinion of the entourage $(P=0.047)$. Positive attitudes of students towards the choice of family medicine were more frequent among participants of the 2nd cycle $(P=0.005)$. Table 3 shows the results of the study of the factors influencing the choice of specialty in family medicine.

3.5. Multivariate Analysis of the Factors Associated with the Choice of Family Medicine. In multivariate analysis, career development ambition and income seemed to be the most powerful factors in the model $(\mathrm{OR}=4.8 ; 95 \% \mathrm{CI}[2.46 ; 9.51]$
TABLE 1: Sociodemographic data and origin of participants.

\begin{tabular}{lcc}
\hline Variable & Numbers & Percentage \\
\hline Gender & 190 & \\
Female & 90 & 67.9 \\
Male & & 32.1 \\
\hline Study's year & \\
First & 30 & 14.6 \\
Second & 38 & 10.7 \\
Third & 25 & 13.6 \\
Fourth & 29 & 8.9 \\
Fifth & 20 & 10.4 \\
Sixth & 62 & 7.1 \\
Seventh & 15 & 22.1 \\
Preparing medical thesis & 13 & 5.4 \\
Interns (1st year) & 7 & 4.6 \\
Interns (2nd year) & & 2.5 \\
\hline Medical school & 88 & \\
Marrakech & 71 & 31.4 \\
Casablanca & 34 & 25.4 \\
Rabat & 31 & 12.1 \\
Fes & 20 & 11.1 \\
Oujda & 19 & 7.1 \\
Agadir & 17 & 6.8 \\
Tangier & 6 & 6.1 \\
Parent is a GP & 7 & 2.1 \\
A sibling is a GP & & 2.5 \\
\hline Marital status & 264 & 9.3 \\
Single & 14 & 0.7 \\
Married & 2 & \\
Divorced & & \\
\hline
\end{tabular}

and $\mathrm{OR}=2.6 ; 95 \% \mathrm{CI}[1.11 ; 6.29]$, resp.) compared to the other studied factors (Table 4).

3.6. Thematic Qualitative Analysis of Verbatim. The aim of the content analysis was to explore better the perceptions towards and the associated factors with positive attitudes regarding the future family medicine. The analysis was done regarding the categories and items below (Table 5).

The participants recognized the essential place of general medicine in healthcare but highlighted the negative image of the general practitioner in the care system. Working conditions and income are important factors to take into consideration for a possible upgrading of this discipline.

"General medicine is as important discipline as the others. The problem lies in the stigmatization of these doctors as well as the flagrant difference in salary between specialists and general practitioners which in my opinion is unfair."

"Being a competent general practitioner is the most intellectually stimulating thing, no specialty deals with all pathologies!"

"Public sector working conditions are getting worse and worse."

"The general practitioner was ridiculed... working conditions with a low income. Whereas the general practitioner has more complicated tasks." 


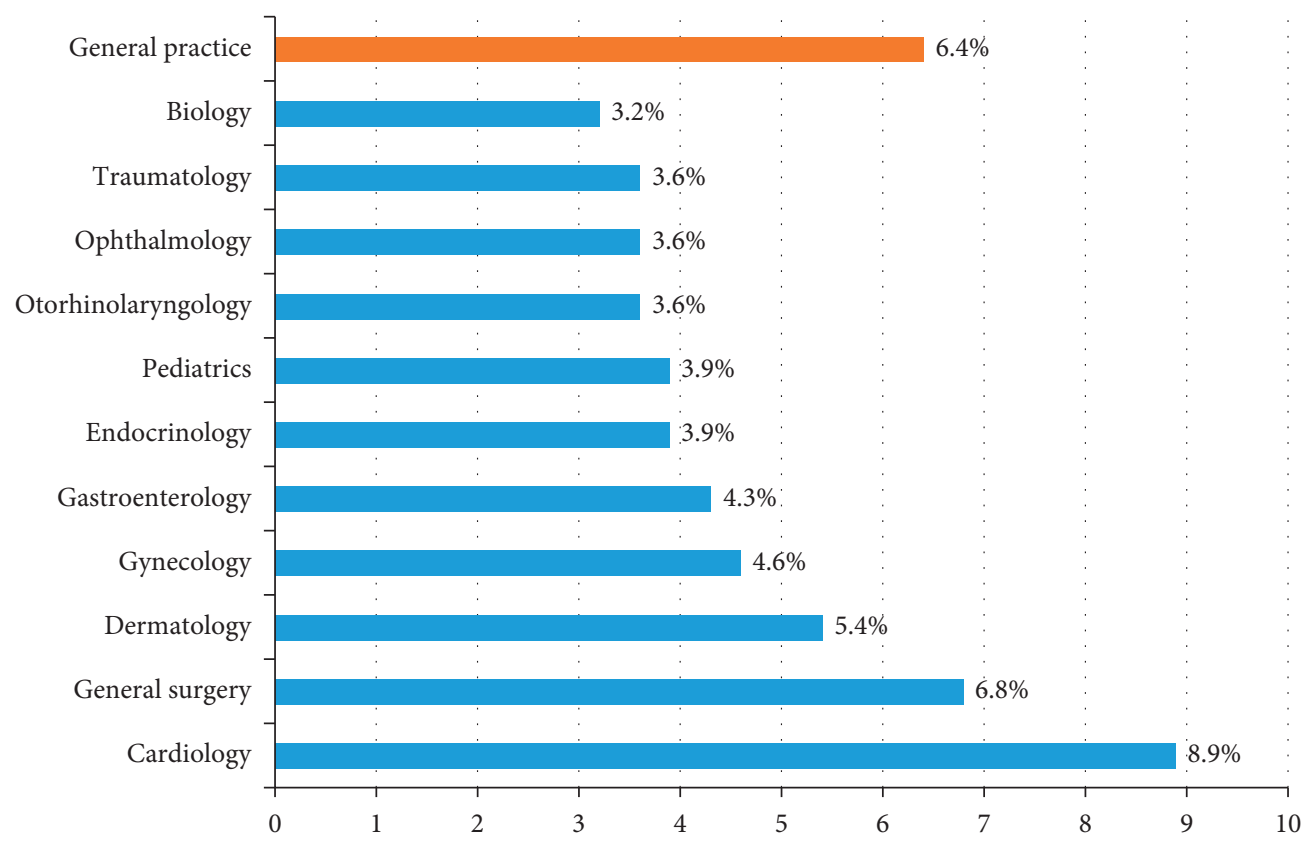

FIGURE 1: Bar chart to show the proportion of responders considering each career option.

TABLE 2: Comparison of Likert scale means of the items according to gender and study level.

\begin{tabular}{|c|c|c|c|c|c|c|c|}
\hline \multirow{3}{*}{ Attitudes } & \multirow{3}{*}{$\begin{array}{c}\text { Total } \\
N=280\end{array}$} & \multicolumn{3}{|c|}{ Cycle of studies score } & \multicolumn{3}{|c|}{ Gender } \\
\hline & & \multicolumn{6}{|c|}{ Mean Likert scale (standard deviations) } \\
\hline & & 1st cycle & $\begin{array}{l}\text { 2nd } \\
\text { cycle }\end{array}$ & $P$ & Female & Male & $P$ \\
\hline \multicolumn{8}{|l|}{ Personal attitude towards general practice } \\
\hline $\begin{array}{l}\text { General practice is a valid discipline like internal medicine or } \\
\text { pediatrics }\end{array}$ & $\begin{array}{c}3.62 \\
(1.197)\end{array}$ & $\begin{array}{c}3.23 \\
(1.16)\end{array}$ & $\begin{array}{c}3.76 \\
(1.18)\end{array}$ & $<0.001$ & $\begin{array}{c}3.66 \\
(1.21)\end{array}$ & $\begin{array}{c}3.56 \\
(1.18)\end{array}$ & 0.394 \\
\hline $\begin{array}{l}\text { Experiences in general medicine are valuable to future doctors, } \\
\text { regardless of their eventual specialty choice }\end{array}$ & $\begin{array}{c}4.19 \\
(0.929)\end{array}$ & $\begin{array}{c}4.13 \\
(0.89)\end{array}$ & $\begin{array}{c}4.21 \\
(0.94)\end{array}$ & 0.338 & $\begin{array}{c}4.24 \\
(0.88)\end{array}$ & $\begin{array}{c}4.09 \\
(1.02)\end{array}$ & 0.347 \\
\hline $\begin{array}{l}\text { General practitioners are particularly capable of providing } \\
\text { comprehensive care }\end{array}$ & $\begin{array}{c}3.53 \\
(1.084)\end{array}$ & $\begin{array}{c}3.34 \\
(1.01)\end{array}$ & $\begin{array}{c}3.60 \\
(1.10)\end{array}$ & 0.055 & $\begin{array}{c}3.57 \\
(1.06)\end{array}$ & $\begin{array}{c}3.44 \\
(1.14)\end{array}$ & 0.363 \\
\hline GPs should spend more time per patient & $\begin{array}{c}3.95 \\
(0.932)\end{array}$ & $\begin{array}{c}4.00 \\
(0.89)\end{array}$ & $\begin{array}{c}3.94 \\
(0.95)\end{array}$ & 0.717 & $\begin{array}{c}4.00 \\
(0.87)\end{array}$ & $\begin{array}{c}3.56 \\
(1.06)\end{array}$ & 0.439 \\
\hline \multicolumn{8}{|l|}{ Comparison to the specialist's work } \\
\hline $\begin{array}{l}\text { The work of a hospital specialist is intellectually more stimulating } \\
\text { than that of general practitioners }\end{array}$ & $\begin{array}{c}3.34 \\
(1.310)\end{array}$ & $\begin{array}{c}3.65 \\
(1.31)\end{array}$ & $\begin{array}{c}3.23 \\
(1.30)\end{array}$ & 0.017 & $\begin{array}{c}3.26 \\
(1.31)\end{array}$ & $\begin{array}{c}3.50 \\
(1.29)\end{array}$ & 0.144 \\
\hline $\begin{array}{l}\text { The work of a specialist working in ambulant care is intellectually } \\
\text { more stimulating than that of general practitioners }\end{array}$ & $\begin{array}{c}3.16 \\
(1.177)\end{array}$ & $\begin{array}{c}3.48 \\
(1.11)\end{array}$ & $\begin{array}{c}3.06 \\
(1.18)\end{array}$ & 0.014 & $\begin{array}{c}3.17 \\
(1.16)\end{array}$ & $\begin{array}{c}3.14 \\
(1.22)\end{array}$ & 0.892 \\
\hline $\begin{array}{l}\text { A specialist (e.g., cardiologist) must earn more money than a } \\
\text { general practitioner }\end{array}$ & $\begin{array}{c}3.52 \\
(1.230)\end{array}$ & $\begin{array}{c}3.80 \\
(1.15)\end{array}$ & $\begin{array}{c}3.43 \\
(1.24)\end{array}$ & 0.026 & $\begin{array}{c}3.43 \\
(1.23)\end{array}$ & $\begin{array}{c}3.71 \\
(1.21)\end{array}$ & 0.062 \\
\hline $\begin{array}{l}\text { General practitioners have deeper relationships with their patients } \\
\text { than specialists }\end{array}$ & $\begin{array}{c}3.24 \\
(1.181)\end{array}$ & $\begin{array}{c}3.14 \\
(1.10)\end{array}$ & $\begin{array}{c}3.27 \\
(1.21)\end{array}$ & 0.456 & $\begin{array}{c}3.16 \\
(1.20)\end{array}$ & $\begin{array}{c}3.40 \\
(1.14)\end{array}$ & 0.132 \\
\hline $\begin{array}{l}\text { I would feel frustrated if I could not obtain a specialty (other than } \\
\text { general practice) }\end{array}$ & $\begin{array}{l}3.60 \\
(1.286)\end{array}$ & $\begin{array}{c}3.86 \\
(1.291)\end{array}$ & $\begin{array}{c}3.51 \\
(1.28)\end{array}$ & 0.026 & $\begin{array}{c}3.61 \\
(1.28)\end{array}$ & $\begin{array}{l}3.59 \\
(1.32)\end{array}$ & 0.947 \\
\hline A general practitioner must enjoy the same prestige as the specialist & $\begin{array}{c}3.62 \\
(1.142)\end{array}$ & $\begin{array}{c}3.61 \\
(1.12)\end{array}$ & $\begin{array}{c}3.63 \\
(1.15)\end{array}$ & 0.895 & $\begin{array}{c}3.63 \\
(1.12)\end{array}$ & $\begin{array}{c}3.61 \\
(1.19)\end{array}$ & 0.912 \\
\hline \multicolumn{8}{|l|}{ Image and prestige of general medicine } \\
\hline $\begin{array}{l}\text { Being in general practice offers the doctor an excellent opportunity } \\
\text { to earn a good living }\end{array}$ & $\begin{array}{c}3.22 \\
(1.040)\end{array}$ & $\begin{array}{c}3.13 \\
(1.01)\end{array}$ & $\begin{array}{c}3.25 \\
(1.05)\end{array}$ & 0.438 & $\begin{array}{c}3.24 \\
(1.031)\end{array}$ & $\begin{array}{c}3.17 \\
(1.063)\end{array}$ & 0.632 \\
\hline Generalists are the most respected members of their communities & $\begin{array}{c}2.58 \\
(1.006)\end{array}$ & $\begin{array}{c}2.72 \\
(0.81)\end{array}$ & $\begin{array}{c}2.54 \\
(1.06)\end{array}$ & 0.073 & $\begin{array}{c}2.52 \\
(1.00)\end{array}$ & $\begin{array}{c}2.71 \\
(1.02)\end{array}$ & 0.207 \\
\hline $\begin{array}{l}\text { Treating acute illnesses is more beneficial than counseling or caring } \\
\text { for patients with chronic illnesses }\end{array}$ & $\begin{array}{c}2.86 \\
(1.219)\end{array}$ & $\begin{array}{c}3.06 \\
(1.17)\end{array}$ & $\begin{array}{c}2.80 \\
(1.23)\end{array}$ & 0.101 & $\begin{array}{c}2.68 \\
(1.20)\end{array}$ & $\begin{array}{c}3.24 \\
(1.19)\end{array}$ & $<0.0001$ \\
\hline $\begin{array}{l}\text { Maintaining health (prevention) is not as interesting to me as } \\
\text { curative treatment }\end{array}$ & $\begin{array}{c}2.25 \\
(1.217)\end{array}$ & $\begin{array}{c}2.46 \\
(1.19)\end{array}$ & $\begin{array}{c}2.18 \\
(1.22)\end{array}$ & 0.048 & $\begin{array}{c}2.02 \\
(1.09)\end{array}$ & $\begin{array}{c}2.74 \\
(1.32)\end{array}$ & $<0.001$ \\
\hline
\end{tabular}


TABle 2: Continued.

\begin{tabular}{|c|c|c|c|c|c|c|c|}
\hline \multirow{3}{*}{ Attitudes } & \multirow{3}{*}{$\begin{array}{c}\text { Total } \\
N=280\end{array}$} & \multicolumn{4}{|c|}{ Cycle of studies score } & \multicolumn{2}{|l|}{ Gender } \\
\hline & & \multicolumn{6}{|c|}{ Mean Likert scale (standard deviations) } \\
\hline & & 1st cycle & $\begin{array}{l}\text { 2nd } \\
\text { cycle }\end{array}$ & $P$ & Female & Male & $P$ \\
\hline $\begin{array}{l}\text { I prefer to spend time dealing with patients' medical problems } \\
\text { rather than their psychosocial problems }\end{array}$ & $\begin{array}{c}2.71 \\
(1.316)\end{array}$ & $\begin{array}{c}2.73 \\
(1.39)\end{array}$ & $\begin{array}{c}2.71 \\
(1.29)\end{array}$ & 0.997 & $\begin{array}{c}2.53 \\
(1.26)\end{array}$ & $\begin{array}{c}3.10 \\
(1.35)\end{array}$ & 0.001 \\
\hline As a doctor, I take more pleasure in talking to patients & $\begin{array}{c}3.74 \\
(0.991)\end{array}$ & $\begin{array}{c}3.90 \\
(0.85)\end{array}$ & $\begin{array}{c}3.68 \\
(1.03)\end{array}$ & 0.169 & $\begin{array}{c}3.78 \\
(0.98)\end{array}$ & $\begin{array}{c}3.64 \\
(1.02)\end{array}$ & 0.302 \\
\hline \multicolumn{8}{|l|}{ Future perspectives } \\
\hline $\begin{array}{l}\text { If I was asked to include the three most fascinating medical } \\
\text { specialties, I would include general medicine }\end{array}$ & $\begin{array}{c}2.92 \\
(1.284)\end{array}$ & $\begin{array}{c}2.52 \\
(1.16)\end{array}$ & $\begin{array}{c}3.06 \\
(1.30)\end{array}$ & 0.003 & $\begin{array}{c}2.94 \\
(1.27)\end{array}$ & $\begin{array}{c}2.89 \\
(1.32)\end{array}$ & 0.762 \\
\hline $\begin{array}{l}\text { The future as a specialist is more attractive than that of the general } \\
\text { practitioner }\end{array}$ & $\begin{array}{c}3.93 \\
(0.981)\end{array}$ & $\begin{array}{c}4.03 \\
(0.96) \\
\end{array}$ & $\begin{array}{c}3.89 \\
(0.99)\end{array}$ & 0.312 & $\begin{array}{c}3.89 \\
(0.98) \\
\end{array}$ & $\begin{array}{c}4.00 \\
(0.98)\end{array}$ & 0.348 \\
\hline \multicolumn{8}{|l|}{ Skills and qualities of a general practitioner } \\
\hline A wide variety of issues affecting all age groups interest me & $\begin{array}{c}3.74 \\
(1.012)\end{array}$ & $\begin{array}{c}3.70 \\
(0.93)\end{array}$ & $\begin{array}{c}3.76 \\
(1.04)\end{array}$ & 0.520 & $\begin{array}{c}3.69 \\
(1.03)\end{array}$ & $\begin{array}{c}3.86 \\
(0.98)\end{array}$ & 0.189 \\
\hline $\begin{array}{l}\text { The care of geriatric patients (elderly subjects) is less interesting for } \\
\text { me }\end{array}$ & $\begin{array}{c}2.78 \\
(1.233)\end{array}$ & $\begin{array}{c}2.54 \\
(1.13)\end{array}$ & $\begin{array}{c}2.86 \\
(1.26)\end{array}$ & 0.084 & $\begin{array}{c}2.68 \\
(1.23)\end{array}$ & $\begin{array}{c}2.98 \\
(1.23)\end{array}$ & 0.076 \\
\hline The quality of medical care provided by GPs is not good & $\begin{array}{c}2.56 \\
(1.184)\end{array}$ & $\begin{array}{c}2.48 \\
(1.15)\end{array}$ & $\begin{array}{c}2.58 \\
(1.20)\end{array}$ & 0.594 & $\begin{array}{c}2.43 \\
(1.17)\end{array}$ & $\begin{array}{c}2.83 \\
1.173\end{array}$ & 0.008 \\
\hline $\begin{array}{l}\text { In case of uncertainty, generalists should not hesitate to consult a } \\
\text { specialist }\end{array}$ & $\begin{array}{c}4.48 \\
(0.851)\end{array}$ & $\begin{array}{c}4.37 \\
(1.02)\end{array}$ & $\begin{array}{c}4.51 \\
(0.79)\end{array}$ & 0.488 & $\begin{array}{c}4.54 \\
(0.70)\end{array}$ & $\begin{array}{c}4.33 \\
(1.09)\end{array}$ & 0.426 \\
\hline Generalist is not as competent as the internist or another specialist & $\begin{array}{c}2.56 \\
(1.234)\end{array}$ & $\begin{array}{c}2.73 \\
(1.24)\end{array}$ & $\begin{array}{c}2.50 \\
(1.23)\end{array}$ & 0.155 & $\begin{array}{c}2.42 \\
(1.17)\end{array}$ & $\begin{array}{c}2.86 \\
(1.32)\end{array}$ & 0.008 \\
\hline $\begin{array}{l}\text { Complex professional training in general practice in many places } \\
\text { and including different specialties is a reason not to choose it as a } \\
\text { career option }\end{array}$ & $\begin{array}{c}2.99 \\
(1.145)\end{array}$ & $\begin{array}{c}3.11 \\
(0.99)\end{array}$ & $\begin{array}{c}2.94 \\
(1.19)\end{array}$ & 0.276 & $\begin{array}{c}2.90 \\
(1.14)\end{array}$ & $\begin{array}{c}3.17 \\
(1.13)\end{array}$ & 0.075 \\
\hline $\begin{array}{l}\text { General practitioner should not take long-term responsibility for } \\
\text { patients with chronic illness alone }\end{array}$ & $\begin{array}{c}3.39 \\
(1.127)\end{array}$ & $\begin{array}{c}3.37 \\
(0.93)\end{array}$ & $\begin{array}{c}3.40 \\
(1.19)\end{array}$ & 0.699 & $\begin{array}{c}3.34 \\
(1.16)\end{array}$ & $\begin{array}{c}3.50 \\
(1.06)\end{array}$ & 0.288 \\
\hline $\begin{array}{l}\text { General practitioner should always consult specialists for patient } \\
\text { management }\end{array}$ & $\begin{array}{c}2.81 \\
(1.208)\end{array}$ & $\begin{array}{c}3.13 \\
(1.23)\end{array}$ & $\begin{array}{c}2.70 \\
(1.18)\end{array}$ & 0.013 & $\begin{array}{c}2.78 \\
(1.21)\end{array}$ & $\begin{array}{c}2.87 \\
(1.20)\end{array}$ & 0.601 \\
\hline $\begin{array}{l}\text { Other health professionals (nurse, medical assistants) should be } \\
\text { integrated into primary care }\end{array}$ & $\begin{array}{c}3.80 \\
(0.997)\end{array}$ & $\begin{array}{c}3.62 \\
(0.99)\end{array}$ & $\begin{array}{c}3.86 \\
(0.99)\end{array}$ & 0.072 & $\begin{array}{c}3.85 \\
(0.97)\end{array}$ & $\begin{array}{c}3.69 \\
(1.06)\end{array}$ & 0.304 \\
\hline $\begin{array}{l}\text { The provision of services by specialized doctors must be } \\
\text { coordinated and controlled by general practitioners }\end{array}$ & $\begin{array}{c}3.35 \\
(1.116)\end{array}$ & $\begin{array}{c}3.23 \\
(1.05)\end{array}$ & $\begin{array}{c}3.39 \\
(1.14)\end{array}$ & 0.207 & $\begin{array}{c}3.34 \\
(1.10)\end{array}$ & $\begin{array}{c}3.37 \\
(1.15)\end{array}$ & 0.766 \\
\hline Epidemiological and preventive research in medicine interests me & $\begin{array}{c}3.31 \\
(1.275)\end{array}$ & $\begin{array}{c}3.15 \\
(1.27)\end{array}$ & $\begin{array}{c}3.36 \\
(1.28)\end{array}$ & 0.272 & $\begin{array}{c}3.27 \\
(1.27)\end{array}$ & $\begin{array}{c}3.38 \\
(1.30)\end{array}$ & 0.510 \\
\hline General practice is based on sound scientific principles & $\begin{array}{c}3.94 \\
(0.902)\end{array}$ & $\begin{array}{l}3.97 \\
(086)\end{array}$ & $\begin{array}{c}3.92 \\
(0.92)\end{array}$ & 0.834 & $\begin{array}{c}3.90 \\
(0.86)\end{array}$ & $\begin{array}{c}4.01 \\
(0.99)\end{array}$ & 0.144 \\
\hline $\begin{array}{l}\text { The psychosocial care by the general practitioner encroaches on the } \\
\text { time to allocate to clinical care }\end{array}$ & $\begin{array}{c}3.24 \\
(1.092)\end{array}$ & $\begin{array}{c}3.13 \\
(1.07)\end{array}$ & $\begin{array}{c}3.28 \\
(1.10)\end{array}$ & 0.328 & $\begin{array}{c}3.24 \\
(1.10)\end{array}$ & $\begin{array}{c}3.24 \\
(1.07)\end{array}$ & 0.919 \\
\hline The introduction of electronic patient records is essential & $\begin{array}{c}4.15 \\
(0.899)\end{array}$ & $\begin{array}{c}4.01 \\
(0.89)\end{array}$ & $\begin{array}{c}4.20 \\
(0.90)\end{array}$ & 0.082 & $\begin{array}{c}4.15 \\
(0.81)\end{array}$ & $\begin{array}{c}4.14 \\
(1.07)\end{array}$ & 0.399 \\
\hline $\begin{array}{l}\text { Respect for a referral system between general practitioner and other } \\
\text { levels of care is essential }\end{array}$ & $\begin{array}{c}4.36 \\
(0.744)\end{array}$ & $\begin{array}{c}4.25 \\
(0.77)\end{array}$ & $\begin{array}{c}4.40 \\
(0.73)\end{array}$ & 0.142 & $\begin{array}{c}4.39 \\
(0.68)\end{array}$ & $\begin{array}{c}4.30 \\
(0.87)\end{array}$ & 0.710 \\
\hline Perceived place of GP in the Moroccan health system & & & & & & & \\
\hline $\begin{array}{l}\text { The Moroccan health system can only work well with general } \\
\text { practitioners as an integral part of the system }\end{array}$ & $\begin{array}{c}4.09 \\
(1.066)\end{array}$ & $\begin{array}{c}3.85 \\
(1.20)\end{array}$ & $\begin{array}{c}4.17 \\
(1.01)\end{array}$ & 0.05 & $\begin{array}{c}4.05 \\
(1.04)\end{array}$ & $\begin{array}{c}4.17 \\
(1.12)\end{array}$ & 0.169 \\
\hline Morocco needs more specialists than generalists & $\begin{array}{c}3.36 \\
(1.202)\end{array}$ & $\begin{array}{c}3.62 \\
(1.29)\end{array}$ & $\begin{array}{c}3.28 \\
(1.16)\end{array}$ & 0.045 & $\begin{array}{c}3.40 \\
(1.15)\end{array}$ & $\begin{array}{c}3.29 \\
(1.30)\end{array}$ & 0.514 \\
\hline Working as a general practitioner in Morocco is unattractive & $\begin{array}{c}3.74 \\
(1.120) \\
\end{array}$ & $\begin{array}{c}3.68 \\
(1.25) \\
\end{array}$ & $\begin{array}{c}3.76 \\
(1.07) \\
\end{array}$ & 0.820 & $\begin{array}{c}3.69 \\
(1.11)\end{array}$ & $\begin{array}{c}3.83 \\
(1.13)\end{array}$ & 0.267 \\
\hline
\end{tabular}

From the participants' point of view, the continuing educations of primary care practitioners as well as the strengthening of skills for the practice of general medicine during undergraduate education are two keys to achieve the goal (creating a residency program or family medicine specialty). The institutionalization of training as a specialty like other specialties will increase the choice of general medicine as a career.
"It is a long-term work to upgrade the general practitioner, who remains, above all, the primary care doctor, in the hope that the new generation of general practitioners will give more value to this noble profession."

"Additional training in general medicine would help me improve my clinical skills and encourage me to choose it as a specialty." 
TABLE 3: Factors associated with career choice of family medicine.

\begin{tabular}{|c|c|c|c|}
\hline \multirow{2}{*}{ Factors } & \multicolumn{2}{|c|}{ Choosing career of family medicine } & \multirow{2}{*}{$P^{*}$} \\
\hline & No $(N=201) n(\%)$ & Yes $(N=77) n(\%)$ & \\
\hline \multicolumn{4}{|l|}{ Gender } \\
\hline Female & $132(65.7)$ & $56(72.7)$ & \multirow{2}{*}{0.163} \\
\hline Male & $69(34.3)$ & $21(27.3)$ & \\
\hline \multicolumn{4}{|c|}{ Parent is GP } \\
\hline No & $191(95.0)$ & $71(92.2)$ & \multirow{2}{*}{0.262} \\
\hline Yes & $10(05.0)$ & $6(7.8)$ & \\
\hline \multicolumn{4}{|c|}{ Study level } \\
\hline 1 st & $60(29.9)$ & $11(14.3)$ & \multirow{2}{*}{0.005} \\
\hline 2nd & $141(70.1)$ & $66(85.7)$ & \\
\hline \multicolumn{4}{|c|}{ Personal experience as a patient in contact with a GP } \\
\hline No & $145(72.1)$ & $42(54.5)$ & \multirow{2}{*}{0.004} \\
\hline Yes & $56(27.9)$ & $35(45.5)$ & \\
\hline \multicolumn{4}{|c|}{ Personal experience as a student in contact with a GP } \\
\hline No & $136(67.7)$ & $32(41.6)$ & \multirow[b]{2}{*}{$<0.0001$} \\
\hline Yes & $65(32.3)$ & $45(58.4)$ & \\
\hline \multicolumn{4}{|c|}{ Ambition of career development } \\
\hline No & $47(23.4)$ & $42(54.5)$ & \multirow{2}{*}{$<0.0001$} \\
\hline Yes & $154(76.6)$ & $35(45.5)$ & \\
\hline \multicolumn{4}{|c|}{ Quality of clinical skills } \\
\hline No & $149(74.1)$ & $42(54.5)$ & \multirow{2}{*}{0.002} \\
\hline Yes & $52(25.9)$ & $35(45.5)$ & \\
\hline \multicolumn{4}{|c|}{ Quality of humans and psychosocial skills } \\
\hline No & $164(82.8)$ & $34(44.2)$ & \multirow{2}{*}{$<0.0001$} \\
\hline Yes & $37(18.4)$ & $43(55.8)$ & \\
\hline \multicolumn{4}{|l|}{ Income } \\
\hline No & $155(77.1)$ & $64(83.1)$ & \multirow{2}{*}{0.176} \\
\hline Yes & $46(22.9)$ & $13(16.9)$ & \\
\hline \multicolumn{4}{|c|}{ Influence of the media (television, radio) } \\
\hline No & $187(93.0)$ & $70(90.9)$ & \multirow{2}{*}{0.354} \\
\hline Yes & $14(7.0)$ & $07(9.1)$ & \\
\hline \multicolumn{4}{|c|}{ Opinion of the entourage (family, friends) } \\
\hline No & $175(87.1)$ & $60(77.9)$ & \multirow{2}{*}{0.047} \\
\hline Yes & $26(12.9)$ & $17(22.1)$ & \\
\hline
\end{tabular}

${ }^{*} P$ value (two percent comparison test using the Fisher's test).

TABLE 4: Results of the multivariate analysis by binary logistic regression of the factors associated with the choice of family medicine as a future specialty.

\begin{tabular}{|c|c|c|c|c|}
\hline \multirow{2}{*}{ Factors } & \multirow{2}{*}{ Odds ratio } & \multicolumn{2}{|c|}{$95 \% \mathrm{CI}$} & \multirow{2}{*}{$P$} \\
\hline & & Lower & Upper & \\
\hline Career development ambition & 4.8 & 2.464 & 9.514 & $<0.001$ \\
\hline Income & 2.644 & 1.111 & 6.292 & 0.028 \\
\hline Experience as a student or patient in contact with GPs & 0.483 & 0.254 & 0.920 & $<0.001$ \\
\hline Study level & 0.404 & 0.172 & 0.950 & 0.038 \\
\hline Entourage opinion & 0.336 & 0.147 & 0.769 & 0.010 \\
\hline Self-perceived skills level (human and psychological) & 0.119 & 0.059 & 0.240 & $<0.001$ \\
\hline Constant $(B=-0.959)$ & 1.614 & & & 0.343 \\
\hline
\end{tabular}

Hosmer and Lemeshow: Chi square $=2.283, P=0.943$.

TABLE 5: The categories of content analysis.

\begin{tabular}{|c|c|c|c|c|}
\hline Categories & Item & Items & Items & Items \\
\hline $\begin{array}{l}\text { Self-perception and } \\
\text { influencing factors }\end{array}$ & Comparison between specialties and GPs & $\begin{array}{l}\text { Influence of opinions: } \\
\text { family, other doctors }\end{array}$ & Income & $\begin{array}{l}\text { Working } \\
\text { conditions }\end{array}$ \\
\hline Recommendations & Improve skills of GPs & $\begin{array}{l}\text { Creation of residency } \\
\text { (continuing educations) }\end{array}$ & + & + \\
\hline
\end{tabular}


"The general practitioner remains the pillar of the health system but we notice that he/she is very devalued (even honestly general practitioners generally have a poor level of skills given the lack of continuing education) I think that the family medicine solution is the best... That way the general practitioner is better supervised and knows better what to do and when to refer patients to the specialist."

"Personally, I think general medicine is very important and attractive, except that it is underestimated by the publicl media and by fellow doctors. What would not encourage me to choose it too is that the general practitioner does not receive additional training when he will have to see pathologies of a few of all specialties."

\section{Discussion}

4.1. Career Choice of Respondents. The majority of respondents wanted to become a medical specialist after the defense of their thesis (obtaining the medical degree). Cardiology and general surgery were the first choices. This can be explained by the prestige of cardiology in our context and the increased demand for these two specialties in private practice with consequently better remuneration. Over $20 \%$ stated that they were considering a career as a GP if the faculty creates the specialty like other countries around the world (family medicine). This percentage falls within the range of results from similar studies in the literature. In some countries like Turkey or Egypt, the reluctance was more marked. Only $4.7 \%$ showed an intention to choose it as a future career according to Alkot et al. in 2015 [11]. In Turkey, it was the least popular specialty with only $0.9 \%(n=7 / 717)$ [12]. The proportion was higher in other sites where family medicine has been established for a longer time such as the United Kingdom (UK). Therefore, among 280 students surveyed in the UK, $40 \%$ said that general practice was an attractive or very attractive career option according to the results of the study by Barber et al. [6]. Our results are close to those reported in China and Canada. According to Zhang et al. in 2016 from 2,714 Chinese students, 19.1\% expressed the desire to choose community work after graduation. This percentage was $20 \%$ according to the Canadian study by Wright et al. $[13,14]$.

\subsection{Attitudes towards General Medicine and Family Medicine.} The results suggest that students have positive attitudes towards general practice as a specialty. The difficulties of recruiting general practitioners and the attitudes of medical students towards the practice of general medicine were studied by many authors around the world (China, England, and Germany) $[6,13,15]$. This, in particular, is following the institutional reforms undertaken in medical education to encourage the choice of career in the primary care. The findings are similar between the different studies. Even if the attitudes towards general medicine are positive, its choice remains variable depending on the context and a certain number of factors [16]. In general, although some students believe that family medicine is attractive, and playing an essential social role, it is regarded as a career of low interest and prestige within the medical profession even in countries where the specialty has been recognized for a long time $[17,18]$. For example, only $10(1.7 \%)$ respondents (5 males and 5 females/591 participants) stated that their first preference was general practice in Greek medical schools [19]. According to Challepah et al., only $13 \%$ of students surveyed in London in 2014 classified general medicine as a career of first choice [20]. The same author concluded that students' exposure to general medicine and primary care research is important.

4.3. Factors Associated with Attitudes and Choice of Family Medicine. All the authors of the studies cited tried to understand what factors were likely to influence the choice of students during and at the end of the curriculum. Through this work, we have attempted to explore these factors in order to propose actions to be implemented that promotes the choice of first-line practice.

Our results highlighted many of the same factors as those found in previous studies that looked at the career choices of medical students, such as the importance of previous exposure to the first line of care during initial studies $[8,21]$. According to Barber et al., 49\% believed that the general culture of their medical school had negatively influenced their vision of general medicine. This highlights the importance of role models during undergraduate education and FM social responsibility [6]. Indeed, our students have a positive attitude towards general medicine, but would refuse to choose it because of the experience linked to general practitioners, and the higher importance and prestige given to specialists in the healthcare system. The initial training is mainly done in contact with specialists at the 3rd level of care in university hospitals. The low attendance of general practitioners and the lack of primary care training would accentuate the negative image of general medicine in our context.

Having visited a primary health care unit as consumers before [11], and the contact with general practice, through student placements and as a patient, are the most significant factor influencing attitudes of final-year medical students towards general practice as a career in New Zealand. Effective positive influences include being welcomed, involved, valued, and given legitimate roles during clinical placements in general practice [22]. This applies to our country, given the absence of specific pedagogical training in supervision for general practitioners in health centers who welcome students during their internship. Over 7 years of training, the transition to the first line is only in the 1 st year for 1 month, in the 2 nd year for 1 month, in the 6th year for 2 months, and in the 7 th year for 2 months. Greater undergraduate exposure to family medicine is needed in order to increase knowledge of the field and influence student career choices [12].

The exposure to GP is greater at the later stages of the curriculum for our students (2nd cycle including 6th and 7th years), which would explain the observed role of the study level on positive attitudes. In fact, $85.7 \%$ of graduate students expressed their desire to choose family medicine as a 
specialty versus $14.3 \%$ for younger students $(P=0.005)$. This factor is known to be associated with more positive attitudes in many publications. Zurro et al. observed a significant increase over the curriculum in the perception of FM as an attractive career choice $(36.7 \%, 41.7 \%$, and $50.2 \%$ in years one, three, and five, resp.) [18]. Henderson et al. suggest that greater exposure of medical students to GPs may positively affect future working relationships and career choice regarding general practice. It also suggests that it is important for providers of undergraduate courses to strive to enhance positive general practice role models [10]. Women also have a more positive attitude towards general medicine than men in the literature [23]. Males were less likely to become GPs (OR 0.31) according to the Australian study by Ward et al. [24]. We explored the influence of gender on the attitudes and perceptions of our respondents. Women have recognized the importance of general medicine in the prevention and treatment of chronic diseases and the consideration of psychosocial aspects. In fact, these are fundamental aspects of distinction in general medicine which adopt a preventive and continuous biopsychosocial approach [4].

Working conditions have a decisive influence on the choice of career in general medicine [25]. On the one hand, this could have a significant impact on the image of the discipline and on the ambition of the students to choose it and on the performance of GPs on the other hand. The poor working conditions in general medicine are leading to growing dissatisfaction among general practitioners; decrease in the prestige of general practitioners due to the modification of personal and professional values and attitudes within society; and a poor representation of general practice as a discipline in the medical curriculum $[7,18]$. Note that the conditions are very different between the public and private sectors in our country, which has created a difference in practices, quality of services in addition to the difference in income between the two. Compared to medical specialists, the career in general medicine currently offers few opportunities for professional development, namely, academic career and research. The lack of concrete and competitive opportunities for career development in primary care is a real obstacle perceived by students. Negative media portrayal of general practice and the attitudes of friends and family have also an influence $[18,22]$. The opinion of those around us seemed to play a role. This is all more pressing when it is a doctor family member who could testify in favor or against general medicine. Among all the factors studied, the possibility of career development and remuneration were the most influential with our students.

According to the literature review by Selva Olid et al. (6 studies in UK, Australia, USA, Japan, Malaysia, and Spain), family medicine is considered as a career of lower interest and prestige. The authors suggest that medical school curricula and exposure to family practice seem to play an important role in this perception [17]. The main reason for not choosing family medicine was inadequate understanding of the specialty according to the study conducted in Ghana [26]. In our context, the revaluation of general medicine via the creation of a specialty (family medicine) like the other countries would be of great contribution in the improvement of its image, better communication around the practice in first line in addition to raising awareness among students which could also increase their choice.

4.4. Context Analysis. General practitioners (GPs) suffer from a devalued status in the health care and service system in Morocco. In the current state of medical training, there is no residency for general medicine. Becoming a GP is therefore a failed choice for the majority of students. But it should be noted that there are several opportunities offered in Morocco in favor of general medicine/family medicine. On the one hand, the directives of His Majesty King Mohammed VI, addressed at the 2nd Conference on Health in 2013, in favor of providing efficient responses, in particular to issues relating to the implementation of constitutional law healthcare, develop the mutual health insurance scheme and reduce disparities between regions to guarantee equitable access to healthcare. The second is the establishment of a commission responsible for reforming medical training with the recognition of the specialty in family medicine. The GPs movement in favor of the promotion of this discipline constitutes a point of strength in addition to the collaboration between faculty members, decision makers, and practitioners as a driving force for change. In Marrakech, there is an example of the project which aims to organize the future training through the development of skills referential, the preparation of internship sites, and the training of internship supervisors. But among the threats to the success of such projects is the persistent absence of a legal framework for GPs as a specific one with the consequences in terms of the remuneration of future family doctors and former GPs practitioners and also the negative perception of the population towards the use of primary care.

We recommended to

institutionalize general practice as academic discipline and create a department of family medicine that offers GPs opportunities to develop academic career and skills offer better quality internships in general medicine expose students to academic and professional models and to positive experiences on the primary care raise awareness among students and the population improve working conditions and remuneration of general practitioners

4.5. Limitations and Bias. Strengths of the study: the number of participants from different medical schools in the country so with the possibility to have a general idea. This is the first study to our knowledge focusing on the attitudes of students in Morocco towards the choice of family medicine which is a possible specialty in the medical curriculum. Electronic data collection has broadened the vision to students outside Marrakech (site for developing a pilot development project for the specialty). The limits are regarding difficulty to explore attitudes and bias of social desirability. 


\section{Conclusion}

The present paper analyzed perceptions of Moroccan student doctors and factors associated with the choice of general medicine as a specialty in order to make recommendations to improve recruitment in general medicine and subsequently prepare the choice of family medicine specialty. The students are the key of the success of the new program in family medicine. Students finish their undergraduate education with a more positive attitude towards general medicine. It seems essential to improve the placement sites in primary care and to increase the frequency of contact with competent GPs in the context of strategies for upgrading general medicine among undergraduate students. Encouraging career development and improving compensation through the creation of a family medicine specialty could be key to the success of a national general medicine development project.

\section{Data Availability}

The data used to support the findings of this study are available from the corresponding author upon request.

\section{Conflicts of Interest}

The authors declare that there are no conflicts of interest regarding the publication of this paper.

\section{Acknowledgments}

Thanks are due to all participating students.

\section{References}

[1] "Les Soins de Santé Primaires," 2019, https://www.who.int/fr/ news-room/fact-sheets/detail/primary-health-care.

[2] WHO, "The 2018 Update Global Health Workforce Statistics," World Health Organization, Geneva, Switzerland, 2019, http://www.who.int/hrh/statistics/hwfstats[http://apps.who. int/gho/data/node.main.HWFGRP_0020?lang=en.

[3] Ressources Humaines De La Santé en Chiffres 2016, 2019, http://drh.sante.gov.ma/Lists/Actualites/Attachments/196/ Ressources\%20Humaines\%20de\%20la\%20Sant\%C3\%A9\% 20en\%20chiffres\%202016.pdf.

[4] W. Europe, "The European Definition of General Practice/ Family Medicine: A Statement from Wonca Europe,” 2005, https://www.woncaeurope.org/gp-definitions.

[5] "Projet de réforme des études médicales, pharmaceutiques et odontologiques (Note de synthèse)," 2019, http://wd.fmpm. uca.ma/fmpm/pedag_eva/reforme/projet_reforme.pdf.

[6] S. Barber, R. Brettell, R. Perera-Salazar, T. Greenhalgh, and R. Harrington, "UK medical students' attitudes towards their future careers and general practice: a cross-sectional survey and qualitative analysis of an oxford cohort," BMC Medical Education, vol. 18, no. 1, p. 160, 2018.

[7] I. Natanzon, D. Ose, J. Szecsenyi, and S. Joos, "What factors aid in the recruitment of general practice as a career? an enquiry by interview of general practitioners," Deutsche Medizinische Wochenschrift 1946, vol. 135, no. 20, pp. 10111015, 2010.
[8] M. Amin, S. Chande, S. Park, J. Rosenthal, and M. Jones, "Do primary care placements influence career choice: what is the evidence?" Education for Primary Care, vol. 29, no. 2, pp. 64-67, 2018.

[9] A. H. Eagly and S. Chaiken, The psychology of Attitudes, Harcourt Brace Jovanovich College Publishers, Orlando, FL, US, 1993.

[10] E. Henderson, A. Berlin, and J. Fuller, "Attitude of medical students towards general practice and general practitioners," British Journal of General Practice, vol. 52, no. 478, pp. 359-363, 2002.

[11] M. M. AlKot, M. A. Gouda, M. T. KhalafAllah, M. S. Zahran, M. M. Kallaf, and A. M. Zayed, "Zamily medicine in Egypt from medical students' perspective: a nationwide survey," Teaching and Learning in Medicine, vol. 27, no. 3, pp. 264-273, 2015.

[12] A. Ozcakir, J. Yaphe, and I. Ercan, "Perceptions of family medicine and career choice among first year medical students: a cross-sectional survey in a turkish medical school," Coll Antropol. Juin, vol. 31, no. 2, pp. 595-600, 2007.

[13] L. Zhang, T. Bossert, A. Mahal, G. Hu, Q. Guo, and Y. Liu, "Attitudes towards primary care career in community health centers among medical students in China," BMC Family Practice, vol. 17, no. 1, p. 75, 2016.

[14] B. Wright, I. Scott, W. Woloschuk, F. Brenneis, and J. Bradley, "Career choice of new medical students at three Canadian universities: family medicine versus specialty medicine," Canadian Medical Association Journal, vol. 170, no. 13, pp. 1920-1924, 2004.

[15] T. Deutsch, S. Lippmann, T. Frese, and H. Sandholzer, "Who wants to become a general practitioner? student and curriculum factors associated with choosing a GP career - a multivariable analysis with particular consideration of practice-orientated GP courses," Scandinavian Journal of Primary Health Care, vol. 33, no. 1, pp. 47-53, 2015.

[16] B. Landström, B. Mattsson, P. Nordin, and C. E. Rudebeck, "The characteristics of general practice and the attractiveness of working as a GP: medical students' views," International Journal of Medical Education, vol. 5, pp. 51-55, 2014.

[17] A. Selva Olid, A. M. Zurro, J. J. Villa et al., "Medical students' perceptions and attitudes about family practice: a qualitative research synthesis," BMC Medical Education, vol. 12, p. 81, 2012.

[18] A. M. Zurro, J. J. Villa, A. M. Hijar et al., "Medical student attitudes towards family medicine in Spain: a statewide analysis," BMC Family Practice, vol. 13, p. 47, 2012.

[19] E. D. Avgerinos, P. Msaouel, G. A. Koussidis, N. C. Keramaris, Z. Bessas, and K. Gourgoulianis, "Greek medical students' career choices indicate strong tendency towards specialization and training abroad," Health Policy, vol. 79, no. 1, pp. 101-106, 2006.

[20] M. Chellappah and L. Garnham, “Medical students' attitudes towards general practice and factors affecting career choice: a questionnaire study," London Journal of Primary Care, vol. 6, no. 6, pp. 117-123, 2014.

[21] D. G. Kassebaum, P. L. Szenas, and M. K. Schuchert, "Determinants of the generalist career intentions of 1995 graduating medical students," Academic Medicine, vol. 71, no. 2, pp. 198-209, 1996.

[22] J. Parker, B. Hudson, and T. Wilkinson, "Influences on final year medical students' attitudes to general practice as a career," Journal of Primary Health Care, vol. 6, no. 1, pp. 56-63, 2014. 
[23] C. Kruschinski, B. Wiese, J. Eberhard, and E. HummersPradier, "Attitudes of medical students towards general practice: effects of gender, a general practice clerkship and a modern curriculum," GMS Zeitschrift Fur Medizinische Ausbildung, vol. 28, no. 1, p. Doc16, 2011.

[24] A. M. Ward, M. Kamien, and D. G. Lopez, "Medical career choice and practice location: early factors predicting course completion, career choice and practice location," Medical Education, vol. 38, no. 3, pp. 239-248, 2004.

[25] T. Lambert, R. Goldacre, F. Smith, and M. J. Goldacre, "Reasons why doctors choose or reject careers in general practice: national surveys," British Journal of General Practice, vol. 62, no. 605, pp. e851-e858, 2012.

[26] A. Essuman, C. Anthony-Krueger, and T. A. Ndanu, "Perceptions of medical students about family medicine in Ghana," Ghana Medical Journal, vol. 47, no. 4, pp. 178-184, 2013. 